\title{
INFRASTRUCTURAL ASSETS PROVISIONING IN CLOUD COMPUTING SYSTEMS
}

\author{
Deepak Kumar Mishra ${ }^{1}$, Vikash Yadav², Niranjan Kumar ${ }^{3}$, Amit Gupta ${ }^{4}$ \\ ${ }^{1}$ Department of IT, AITM, Lucknow, India \\ ${ }^{2}$ Department of CSE, HBTI, Kanpur, India \\ ${ }^{3}$ Deptt of CS, AITM, Lucknow, India \\ ${ }^{4}$ Department of IT, AITH, Kanpur, India
}

\begin{abstract}
This paper presents a study on cloud computing infrastructure and hardware resources used. Using cloud computing environment, clients can develop their own services making accessible arrangement of virtual machines. Such methodology can be used in infrastructural assets provisioning and balancing load in data centers. The provisioning of Infrastructural asset can help in implementation of data parallelisms and can also handle user requests more effectively. This paper presents study on different parameters which customizes data center federated services on cloud optimizations platform.
\end{abstract}

Keywords-Cloud Computing, Cloud Assets, Cloud Suppliers, Virtual Machine (VM) and Cloud Service in Data Centers.

\section{INTRODUCTION}

Cloud computing is an extensive scalable computing paradigm that provide appropriate figuring worldview. Infrastructural assets for any business enterprises are accessible to clients (called cloud service customer) by means of the Internet. Computing assets, e.g., network bandwidth, executing Speed, storage, programming, are spoken to cloud customers as the open utility administrations. Infrastructure-as-a-Service (IaaS) is service Model that provides access to computing resource in a virtualised environment. The virtualization innovations can be utilized to give assets to cloud purchasers. The customers can indicate the obliged programming software stack, e.g., working frameworks and applications; then bundle all of them together into virtual machines (VMs). The resources prerequisite of VMs can likewise be balanced by the customers. At long last, those VMs will be outsourced to host in processing environment worked by outsider locales claimed by cloud suppliers. Cloud supplier must assure its customer for Quality of Services (QoS) that are delivered for running VMs. Cloud assets are provided by its supplier and it has to be issued on the Total Cost of Ownership (TCO) that shows how cloud user can be upgraded or degraded to premium/general packages. This may also affect Return on Investment (ROI) by any organization.

Cloud computing is a large scale appropriate processing worldview in which a pool of figuring assets is accessible to clients (called cloud customers) by means of the Internet. Figuring assets, e.g., preparing force, stockpiling, programming, and system transmission capacity, are spoken to cloud shoppers as the open utility administrations. Infrastructure-as-a-Service (IaaS) is a computational administration show broadly connected in the distributed computing worldview. In this model, virtualization advancements can be used to give assets to cloud buyers. The customers can determine the obliged programming stack, e.g., working frameworks and applications then bundle every one of them.

With the reservation facility, the cloud shoppers can save the assets ahead of time. In the same manner, the underprovisioning issue can happen when the saved assets are not able to completely take care of the demand because of its vulnerability. Despite the fact that this issue can be unraveled by provisioning more assets with on-interest arrangement to fit the additional interest, the high cost will be brought about because of more extravagant cost of asset provisioning with on-interest arrangement. Then again, the over provisioning issue can happen if the held assets are more than the genuine interest in which a piece of an asset pool will be underutilized. It is essential for the cloud customers to minimize the aggregate expense of asset reducing so as to prove the on-interest expense and over-subscribed expense of under provisioning and over provisioning. To accomplish this objective, the ideal available asset administration is the discriminating issue. In this paper, we analyze the infrastructural asset that could be provisioned over issues for scalability, vulnerability and adaptability in distributed computing environment. It is our encouragement to investigate such study on available provisioning for infrastructural asset in cloud computing system. Specifically, an optimization of cloud resource provisioning (OCRP) calculation is survey to minimize the aggregate expense for infrastructural provisioning assets in a sure time period. This ideal choice came through by figuring and taking care of a stochastic whole number programming issue with multistage plan of action. 
The real commitment of this paper reside in the experimental examination which can be outlined as follows: The optimization cloud resource provisioning (OCRP) calculation, which is studied for the virtual machine administration. The streamlining plan of stochastic for all things that have been considered in TCO of asset provisioning in cloud computing environments. The definition considers different provisioning stages with interest and value vulnerabilities. The arrangement techniques in light of Benders disintegration and test normal estimate calculations are utilized to comprehend the advancement definition in an effective manner. The execution assessment is performed which uncover the significance of ideal registering of resource provisioning. The experimental examination among infrastructural asset and alternate methodologies is likewise displayed. The experimental investigation will be helpful to the cloud buyers (e.g., association and organization) for the administration of virtual machines in distributed computing environment. The studies on infrastructural assets have been analyzed, which encourage the selection of distributed computing of the clients as it can decrease the expense of utilizing figuring asset fundamentally. Related works are investigated in Section 2. The cloud data centers service model distributed computing environments are depicted in Section 3.

\section{RELATED WORK}

Accessible asset provisioning alternatives are discussed in [5]. The asset provisioning methods in appropriate frameworks are intended to be in an engineering outline of on-interest administration for lattice registering has been studied and surveyed. In a profile-based way to deal with catch master's information of scaling applications was also studied in which additional requested assets can be all the more productively provisioned. The goal is to address instability of assets accessibility. In twofold whole number system to amplify incomes and use of asset suppliers was defined. Be that as it may not consider instability of future buyer requests. In advancement system the asset provisioning was produced.

This structure considered various customer QoS classes under vulnerability of workloads (e.g., requests of registering assets). The entry example of workloads is assessed by utilizing internet gauging systems. In heuristic technique for administration reservation was proposed. Expectation of interest was performed to characterize reservation costs. In K-closest neighbours calculation was connected to foresee the interest of assets. Interestingly, our work determines that requests are given as likelihood appropriations. Also, the value contrast in the middle of reservation and on-interest arrangements were not considered in all works in writing. As virtualization is a center innovation of distributed computing, the issue of virtual machine arrangement (VM situation) gets to be pivotal, the merchant based structural planning and calculation for relegating VMs to physical servers were created. In an asset oversee both asset provisioning and VM position was proposed. In methods of VM situation and combination which influence min-max and shares components gave by hypervisors were investigated. In a dynamic combination instrument taking into account limitation writing computer programs were created. This combination instrument was the starting point mainly intended for homogeneous bunches. Be that as it may, hetero-geneity which is regular in a numerous cloud supplier environment was disregarded. In addition did not consider vulnerability of future requests and costs.

In an element VM situation was proposed. Be that as it may, the arrangement in is heuristic-based which can't promise the ideal arrangement. Stochastic programming has been created to unravel asset arranging under vulnerability in different fields, e.g., generation arranging, monetary administration, and scope quantification.

For instance, in, the creators connected the stochastic programming methodology for arrangement of electrical force era and transmission line development while a few instabilities influencing the arrangement were considered. It is demonstrated that stochastic writing computer programs is the promising scientific apparatus which has the capacity address the ideal choice making in the stochastic environment. Nevertheless, to the best of our insight, the utilization of stochastic programming for processing asset procurement has never been contemplated.

Propelled by this past work, we present the OCRP calculation in this paper which accomplishes numerous enhancements. Initially, the parameters which influence infrastructural asset for cloud computing system, is discussed into the numerous stage plans. Second, the diverse ways to deal with the arrangement of figuring asset provisioning are considered. At last, the execution assessment is stretched out to consider different sensible situations.

\section{CLOUD SERVICE DATA CENTERS}

It is characteristic to inquire as to why existing answers for the endeavor server farm does not work for cloud administration server farms. Most important thing is that the main expense in the endeavor is operational Staff. In the server farm, such expenses are so little (under $5 \%$ because of mechanization), that we securely overlook them from Table 1. In a well-run undertaking, a regular proportion of IT staff individuals to servers are 1:100. Mechanization is incomplete and Human mistake is the reason for a huge division of execution, creating problems In cloud administration server farms. Computerization is an obligatory Requirement of scale and it is needed to be a foundational standard of outline. In a well run server farm, a regular proportion of staff individuals to servers is Automated, Recovery-arranged processing systems adapt achievement completely to the lion's share Of issues that emerge. There are extra contrasts between the venture and the cloud administration server farm situations including:

\subsection{Large Economies of Scale}

The measure of cloud scale server farms (some now drawing nearer 100,000 separates) shows an opportunity to influence economies of scale not exhibit in the endeavor server farms; 
however the forthright expenses are high. Scale Out. Endeavors regularly advance for physical space and number of gadgets, uniting Workload onto a little number of highvalue "scale-up" equipment gadgets and servers. Cloud resources management server farms "scale-out" disseminating workload over extensive quantities of minimal effort servers and equipment. That said, undertakings are additionally moving towards the cloud.

\subsection{Types of Cloud Service Data Centers}

Numerous cloud administration server farms today may be termed as server farms, having on the request of many thousands or more servers drawing several Mega-Watts of force at top. Enormous Data investigation applications (e.g., figuring the web inquiry record) are a characteristic fit for a super Data focus, where a few issues require tremendous measures of quick RAM, others oblige Massive numbers of CPU cycles, and still others oblige huge circle I/O bandwidth. These issues regularly call for broad correspondence.

\subsection{Reducing These Costs}

In what capacity can server farm systems and frameworks help to raise usage, and tackle the problems recorded previously? A key component of the arrangement is dexterity: the capacity to progressively develop and shrink assets to take care of demand and to draw those assets from the most ideal area. Today, the system remains as an obstruction to spryness and expands the discontinuity of assets that prompts low server usage. Segment 3 depicts the issues and the properties we look for, in its answer.

\section{EXPERIMENTAL RESULTS}

This paper experiments the several parameters on cloud server and its data centre configuration aspects. Parameters like number of request/ Hrs, Data Size/ Request (Byte), Peak hour's duration in User Base (UB) panel - were settled up. For Data Centers parameters like - Storage (in MB), Memory (RAM), Number of processors, available Band Width, Virtual Machine Model (VMM), processor speed: were analyzed.

We try to focus on loading scheme for seven Data Centers (DC) with different equipment on different performance scheme.

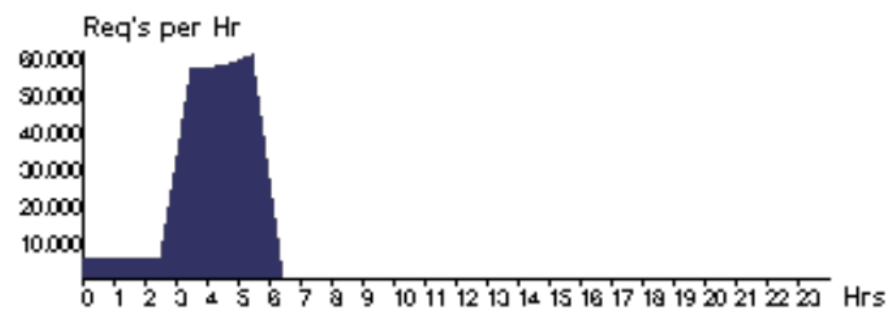

Fig 1: Data Centre 1 (DC 1) loading scheme

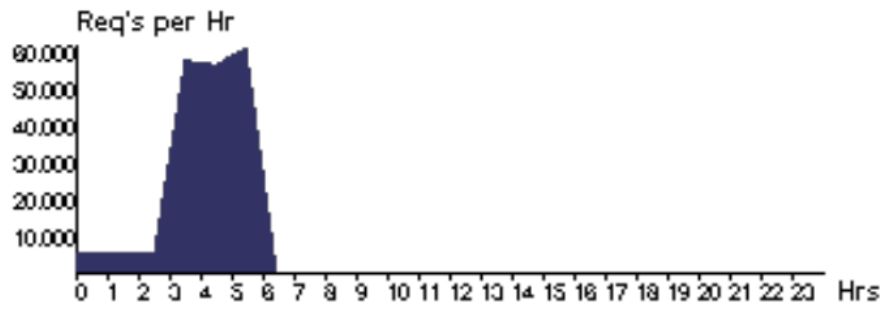

Fig 2: Data Centre 2 (DC 2) loading scheme

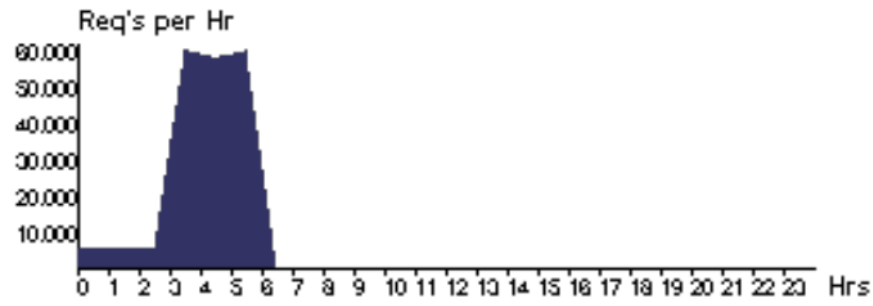

Fig 3: Data Centre 3(DC 3) loading scheme

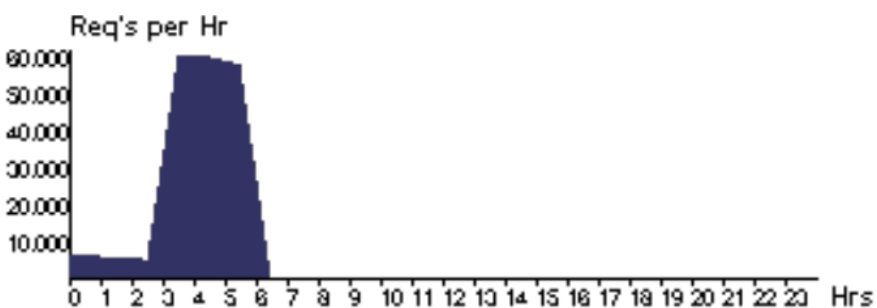

Fig 4: Data Centre 4 (DC 4) loading scheme

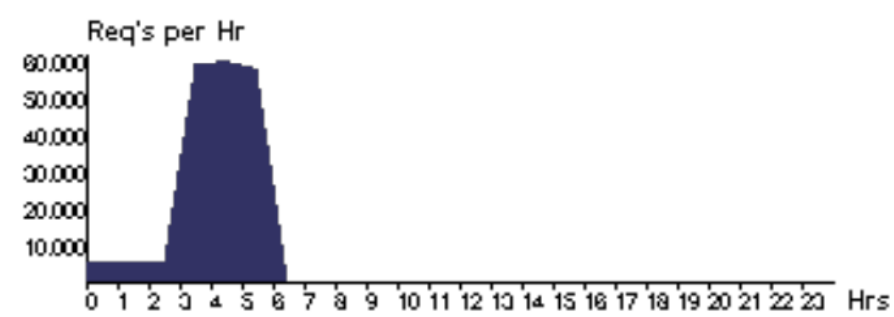

Fig 5: Data Centre 5 (DC 5) loading scheme

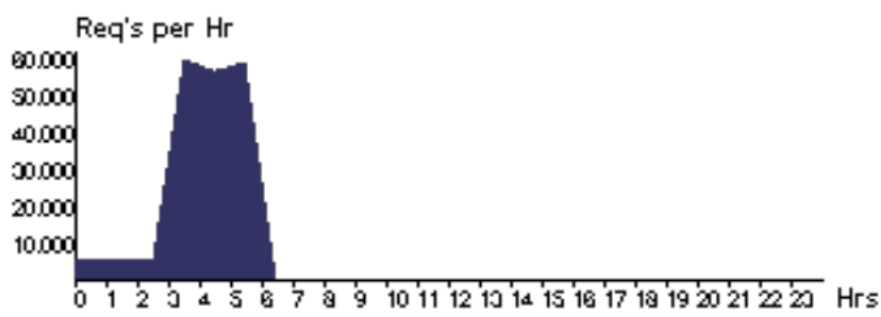

Fig 6: Data Centre 6 (DC 6) loading scheme

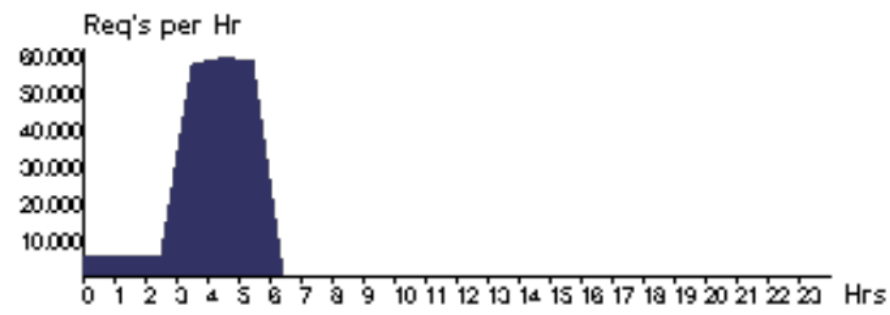

Fig 7: Data Centre 7 (DC 7) loading scheme 


\section{CONCLUSION}

This paper focuses on the study and behavior of Data Centers parameters that have been used in this study. If available asset for any particular Data Center is equipped with rich infrastructural resources, then overall performance to Cloud computing system could be improved. But we can't say, if we highly equipped any data center with resources more than its requirement, then its resources could be under loaded that may lead to deficient ROI.

\section{REFERENCE}

[1] Y. Jie, Q. Jie, and L. Ying, "A Profile-Based Approach to Just-in Time Scalability for Cloud Applications", In Proc. of IEEE International Conference Cloud Computing (CLOUD 09), 2009.

[2] S. Chaisiri and B. Lee, "Optimization of Resource Provisioning Cost in Cloud Computing", In IEEE Transaction on Service Computing, Vol.5, No.2, 164177.

[3] Cloud Servers Pricing by Rackspace Cloud Computing and Hosting, 2013, Available: http://www.rackspace.com/cloud/public/servers/pricin g/

[4] R. Moreno-Vozmediano, R. S. Montero, and I. M. Llorente, "IaaS Cloud Architecture: From Virtualized Datacenters to Federated Cloud Infrastructures", Computer, vol. 45, no. 12, pp. 65-72, Dec., 2012.

[5] R Buyya, R Ranjan, and R. Calheiros, "Intercloud: Utility-oriented federation of cloud computing environments for scaling of application services", Algorithms and architectures for parallel processing, pp.13-31, 2010

[6] L. Badger, T. Grance, R. Patt-Corner, and J. Voas, "Draft cloud computing synopsis and recommendations", NIST Special Publication, 800:146, 2011

[7] R. Van den Bossche, K. Vanmechelen, and J. Broeckhove, "CostOptimal Scheduling in Hybrid IaaS Clouds for Deadline Constrained Workloads", Cloud Computing (CLOUD), 2010 IEEE 3rd International Conference on , pp.228,235, 5-10, July 2010.

[8] B. Javadi, J. Abawajy, and R. Buyya, "Failure-aware resource provisioning for hybrid Cloud infrastructure", Journal of Parallel and Distributed Computing, Vol. 72(10), pp.1318-1331, October 2012.

[9] J. Lucas-Simarro, R. Moreno-Vozmediano, R. Montero, and I. Llorente, "Scheduling strategies for optimal service deployment across multiple clouds", Future Generation Computer Systems, vol.29, no. 6, pp.1431- 1441, August 2013.

[10] D. Breitgand, A. Marashini, and J. Tordsson, "Policydriven service placement optimization in federated clouds,"' IBM Research Division, Tech. Rep, 2011. 2011.

[11] C. Vecchiola, R. Calheiros, D. Karunamoorthy, and R. Buyya, "Deadline-driven provisioning of resources for scientific applications in hybrid clouds with
Aneka", Future Generation Computer Systems, vol. 28, no. 1, pp.58-65, January 2012.

[12] P. Wright, Y. L. Sun, T. Harmer, A. Keenan, A. Stewart, A., and R. Perrott, "A constraints-based resource discovery model for multi-provider cloud environments", Journal of Cloud Computing: Advances, Systems and Applications, vol. 1, no. 1, 2012.

[13] R. Calheiros, A. Toosi, C. Vecchiola, and R. Buyya "A coordinator for scaling elastic applications across multiple clouds", Future Generation Computer Systems Vol.28(8), pp.1350-1362, 2012.

[14] S. He, L. Guo, M. Ghanem and Y. Guo, "Improving resource utilisation in the cloud environment using multivariate probabilistic models," IEEE International Conference on Cloud Computing, 2012.

[15] M. A. Adnan, R. Sugihara and R.K. Gupta, "Energy efficient geographical load balancing via dynamic deferral of workload," IEEE International Conference on Cloud Computing, 2012

[16] K. Halder, U. Bellur and P.Kulkarni, "Risk aware provisioning and resource aggregation based consolidation of virtual machines," IEEE International Conference on Cloud Computing, 2012. 